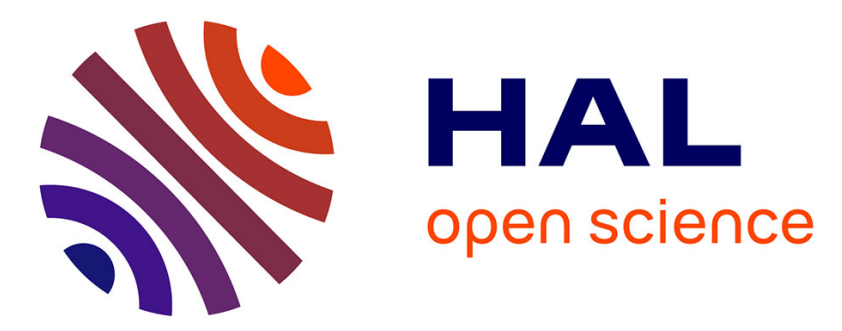

\title{
Inverse Dynamics Model-based Shape Control of Soft Continuum Finger Robot Using Parametric Curve
}

Steeve Mbakop, Gilles Tagnes, Marc-Henri Frouin, Achille Melingui, Rochdi Merzouki

\section{- To cite this version:}

Steeve Mbakop, Gilles Tagnes, Marc-Henri Frouin, Achille Melingui, Rochdi Merzouki. Inverse Dynamics Model-based Shape Control of Soft Continuum Finger Robot Using Parametric Curve. IEEE Robotics and Automation Letters, 2021, 6 (4), pp.8053 - 8060. 10.1109/LRA.2021.3101874 . hal03339777

\section{HAL Id: hal-03339777 https://hal.science/hal-03339777}

Submitted on 9 Sep 2021

HAL is a multi-disciplinary open access archive for the deposit and dissemination of scientific research documents, whether they are published or not. The documents may come from teaching and research institutions in France or abroad, or from public or private research centers.
L'archive ouverte pluridisciplinaire HAL, est destinée au dépôt et à la diffusion de documents scientifiques de niveau recherche, publiés ou non, émanant des établissements d'enseignement et de recherche français ou étrangers, des laboratoires publics ou privés. 


\title{
Inverse Dynamics Model-based Shape Control of Soft Continuum Finger Robot Using Parametric Curve
}

\author{
Steeve Mbakop ${ }^{1,2}$, Gilles Tagnes ${ }^{1,2}$, Marc-Henri Frouin ${ }^{3}$, Achille Melingui ${ }^{4}$ and Rochdi Merzouki ${ }^{1}$
}

\begin{abstract}
Soft continuum robots are more and more used in various applications due to the multiple advantages offered by the properties of hyper-elastic soft materials, such as resilience, flexibility, etc. Despite all these essential properties that make the soft robots good candidates for some real-life needs (form enclosure grasping, obstacle-free navigation, etc.), controlling their shape remains challenging because of their hyper-redundancies. This paper investigates an inverse dynamics model-based shape control of soft continuum robots in the presence and absence of external efforts. To that end, modeling based on Pythagorean Hodograph (PH) curves with prescribed lengths is combined with EulerBernoulli modeling to reconstruct the robot shapes and then calculate the actuator inputs in the case of external efforts. Thus, a relationship between the actuator inputs and the $\mathbf{P H}$ control points is obtained. With the above strategy, the actuator inputs can be computed accordingly, and therefore, real-time shape control of the soft robots for various tasks becomes possible. The results of the proposed approach are validated both numerically and experimentally using two classes of soft continuum robots - a Fluidic Elastomeric Actuator (FEA), describing a 2D soft finger robot, with a single and multiple phalanges, and a 3D continuum manipulator, namely the Compact Bionic Handling Assistant (CBHA).
\end{abstract}

Index Terms-Soft Robot Applications, Modeling, Control, and Learning for Soft Robots, Grasping.

\section{INTRODUCTION}

Recently, the features of soft robots have helped to overcome the difficulties faced by rigid robots in soft and adaptive interactions with external dynamic environments. The soft robots, also called soft actuators, are made of compliant materials [1]. To control their shape, an accurate model that maps the actuation inputs (physical inputs) to the geometric shape of the robot should be obtained. This issue has been investigated by Mochiyama et al. [2].

Manuscript received: February, 24, 2021; Revised June, 1, 2021; Accepted July, 4, 2021

This paper was recommended for publication by Editor Kyu-Jin Cho upon evaluation of the Associate Editor and Reviewers' comments. This work was supported in part by the foundation i-Site, the Regional Council Hauts-deFrance and in part by the company Nyrio.

${ }^{1,2}$ Steeve Mbakop and Gilles Tagne are with CRISTAL, CNRS UMR 9189 and JUNIA, 2 Rue Norbert Segard, 59046 Lille, France steeve.kamga-mbakop@junia.com, gilles.tagnedjunia.com

${ }^{3}$ Marc-Henri Frouin is with Niryo, 165 Avenue de Bretagne, 59000 Lille, France mh. frouin@niryo.com

${ }^{4}$ Achille Melingui is with GET. Department, University of Yaoundé 1, Yaoundé 8390, Cameroon achille.melingui@gmail.com

${ }^{1}$ Rochdi Merzouki is with CRISTAL, CNRS UMR 9189, University of Lille, 59655 Villeneuve d'Ascq, France rochdi.merzouki@univ-lille.fr

Digital Object Identifier (DOI): see top of this page.
In a recent work [3], a model-based shape control using a finite order polynomial curvature method is proposed for Soft Continuum Robots kinematics modeling. Later in [4], a topology reduction for robot kinematics with singularities-free has been developed to control soft robot shape dynamics. An extension to external interactions with high accelerations has been studied in [5]. The issues related to infinite Degrees-ofFreedom (DoFs) using a finite dimension reduction approach have also been addressed.

Soft continuum robot dynamics with space motions have been discussed in the recent literature. Reference [6] introduced a Reduced-Order Model (ROM) for continuum robots dynamics control based on the Principle of Virtual Work (PVW). An approximate series-solution has been used for the kinematics while reconstructing the robot shape by fitting the Lagrange polynomial function at particular points. Later, the author [7] has provided quantitative studies by comparing ROM-based techniques with some other discretized lumped systems. The advantages of ROM techniques might be highlighted, such as the balance between precision and computational time. Dynamics modeling based on CosseratRod has been suggested with the use of Magnus series solutions for dimension reduction purposes [8]. Polynomial fitting techniques have also been used in that case for the shape kinematics reconstruction. Ref. [9] also took benefits from the Cosserat-Rod modeling technique to minimize the set of Ordinary Differential Equation (ODE). The authors succeeded in improving the computing time of the robot dynamics modeling, thanks to a new reduced inverse Newton-Euler algorithm. However, the real-time implementation of the shape controller remains difficult if the relationship between the actuator inputs and the robot's geometric shape is not established. The contribution of this work regarding the current literature is the calculation of the actuator inputs of soft robots based on the shape described by a polynomial parametric curve.

For the shape kinematic modeling of soft continuum robots, parametric curves-based techniques have been recently explored. Reference [10] has solved the kinematics of continuum robots using a $\mathrm{PH}$ curve to reconstruct the overall robot shape. Also, in [11] and [12], a reduced number of the curve control points is highlighted for the kinematics computation. Later in [13], cubic Hermite spline curves have been suggested for accurate shape kinematic computation of Fluidic Elastomeric Actuators (FEA). A recent investigation [14] has proved that the Euler parametric curve is accurate for complex shape 
modeling. Despite the advantageous features of all these parametric curve techniques in terms of accuracy, computing time, and simplicity, their parameters (control points) keep virtual and are not linked to actuation inputs.

In this paper, to deal with complex shapes static reconstruction, we propose a model-based inverse dynamics that uses Euler-Bernoulli's 3D beam modeling coupled with the parametric kinematic $\mathrm{PH}$ curves while taking external efforts into account. The proposed method enables the calculation of actuator inputs, which are used to reconstruct the desired shape of a continuum FEA robot. The shape control is obtained by optimal positioning of the $\mathrm{PH}$ control points, which are calculated as a function of the actuator inputs.

The paper is organized as follows: The second section presents the 3D modeling for FEAs. The third section elaborates the methodology for calculating the actuation inputs of FEAs from the desired shape. In the fourth section, we present the material used and the validation method. A set of numerical and experimental results is presented in the fifth section. The conclusion and future works are finally discussed in the last section.

\section{Euler Bernoulli Modeling for Fluidic Elastomeric Soft Finger Robots}

This section aims to model in 3D the FEA dynamics, based on Euler-Bernoulli's (EB) theory, where a relationship between the curvature of the soft actuator can be established with the actuator inputs. This is to control adaptively the shape of the FEA in presence of external efforts. The curvature can be constant or variable. For the latter, a series of constant curvatures are considered to simulate the variable curvature of a soft actuator. In the case of soft fingers, it implies the consideration of one or multiple actuators described by phalanges, where each phalange is modeled by a constant curvature. In the end, $\mathrm{PH}$ curves are used to reconstruct kinematically the shape of the FEA, depending on the curvature, which is also dependent on actuator inputs.

\section{A. $3 D$ modeling of FEA}

The Euler-Bernoulli theory enables describing the influence of the external efforts on a beam of soft actuators. In the case of FEAs, the dynamics of the bending can be observed by varying the actuator inputs (air pressure) trapped inside the chamber.

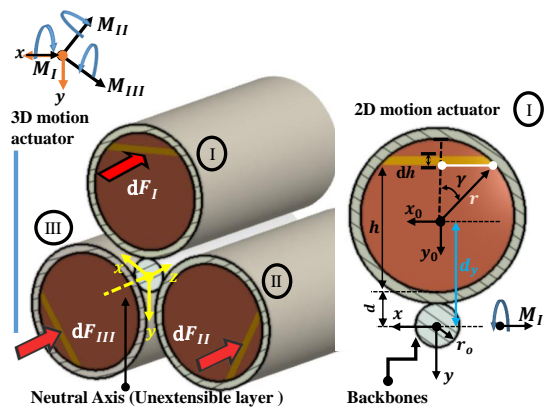

Fig. 1: FEA concept and sizing
Considering the given FEA concept of Fig. 1, the normal force generated by the actuator input, namely air pressure, along the horizontal line of the actuator, being at a distance $h$ from the bottom layer, is given as follows:

$$
\mathrm{d} F_{I}=\left(2 r P_{i n} \sin \gamma\right) \mathrm{d} h,
$$

where $P_{i n}, r$, and $\gamma$ denote respectively the actuator input of air pressure, the radius of the cross section for the soft actuator, and the angle of an infinitesimal distance located on the cross section. $\mathrm{d} F$ is the infinitesimal normal force acting on the cross section due to the air pressure along the infinitesimal distance $\mathrm{d} h$.

Assuming one controlled actuator input $P_{i n}$ for a given actuator, following (1), the bending moment $M_{e}$ (Fig. 2) can be expressed as a function of $P_{i n}$ and a geometric parameter $\Psi$ :

$$
M_{e}=\int(h+d) \mathrm{d} F=\Psi P_{i n} .
$$

However, the deviated bending issued from the combined action of end moments (Fig. 1) should be considered for the 3D motion :

$$
M_{e}=\left[\begin{array}{lll}
M_{e x} & M_{e y} & M_{e z}
\end{array}\right]^{T} .
$$

From Fig. 1, the following is obtained:

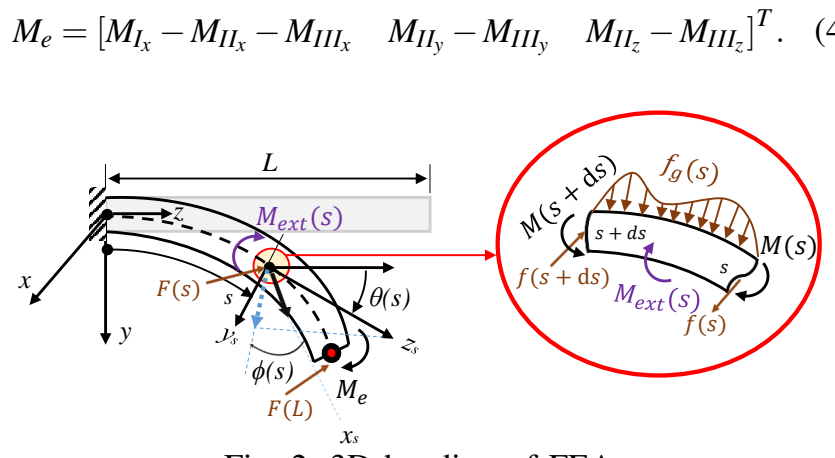

Fig. 2: 3D bending of FEA

\section{B. Flexural stiffness modeling}

The ability of FEA to exhibit a given bending behavior depends on some of their intrinsic properties that take into account both micro and macro structures, called the flexural stiffness $(E I)$. The microstructure is represented with $E$, the elastic Young Modulus for material properties. The macrostructure is described by $I(s)$, representing the local static moment of inertia. For the particular case of a circular crosssection actuator $\left(I_{y_{0}}(s)=I_{x_{0}}(s)\right)$, the local static moment of inertia can be calculated using Huygens-Steiner theorem as follows:

$$
I_{x}(s)=I_{x_{0}}(s)+A_{s}(s) d_{y}^{2},
$$

where $I_{x_{0}}(s)$ represents local quadratic moment of inertia relative to $x_{0}$ in the $y_{0} z_{0}$-frame. During the actuation, the flexural stiffness might increase because of the additional stiffness induced by the compressed fluid pressure. In order to consider this aspect, the following modeling has been considered [15] :

$$
(E I)_{e q_{x}}=\left(E+P_{\text {in }}\right) I_{x}(s) .
$$




\section{Soft actuator curvature modeling}

In the following development, it is assumed that the soft finger length remains constant. For modeling purposes, a soft finger can be viewed as a thin cantilever 3D beam. The FEA is supposed to be subject to an end moment as stated in [16]. Let us consider a thin cantilever beam of length $L$ subjected to a moment $M_{e}$, which is applied at the free end of the beam, as shown in Fig. 2.

We denote $s(0 \leq s \leq L)$ as the curvilinear coordinate along the axial line, measured from the clamped end and $p(s)$ its Cartesian position. Also, let us define $\boldsymbol{\theta}(s)$ and $\phi(s)$, respectively, the polar and the azimuthal positive angles (relative to $x$-axis) of the tangent $p^{\prime}(s)$ to the neutral axis at point $s$ (Fig. 2). In addition, let $M, f$ denote the bending moment and the internal forces at the location $s$, respectively. The external forces and moments are denoted by $F$ and $M_{\text {ext }}$, respectively. The effects of gravitational actions on the structure are described with $f_{g}$, while $g$ denotes the gravity.

The dynamics of the structure yields [17] :

$$
\begin{aligned}
& \mathrm{d} F(s)+f(s)=\rho \ddot{p}(s), \\
& \mathrm{d} M(s)+p^{\prime}(s) \times F(s)+M_{\text {ext }}(s)=J \Theta ̈(s) .
\end{aligned}
$$

where $\Theta(s)=\left[\begin{array}{lll}\theta(s) & \phi(s) & 0\end{array}\right]^{T}$ denotes the rotation angle given in polar form $J=\operatorname{diag}\left(J_{x_{s}}, J_{y_{s}}, J_{z_{s}}\right)$ the quadratic moment of inertia, and $\rho$ is the linear mass distribution. The bending moment that acts at each point of the beam can also be described thanks to EB approach:

$$
\kappa(s)=\left[\begin{array}{ll}
\frac{M_{x_{s}}(s)}{(E I)_{e q_{x_{s}}}} & \frac{M_{y_{s}}(s)}{(E I)_{e q_{y_{s}}}}
\end{array}\right]^{T}, \quad \tau(s)=\frac{M_{z_{s}}(s)}{(E I)_{e q_{z s}}} .
$$

$\kappa(s)$ and $\tau(s)$ denote the local curvature and the local torsion of the FEA, respectively. However, the twisting motion will be neglected $(\tau=0)$ in the remaining of the proposal. The boundary conditions read:

$$
\left\{\begin{aligned}
\left.p(s)\right|_{s=0} & =0 \\
{[\theta(s) \quad \phi(s)]_{s=0} } & =0 \\
\left.F(s)\right|_{s=L} & =F_{e}, \\
{\left[\theta^{\prime}(s) \quad \phi^{\prime}(s)\right]_{s=L} } & =\left[\frac{M_{e_{x}}}{(E I)_{e q_{x_{s}}}} \quad \frac{M_{e_{y}}}{(E I)_{e q_{y_{s}}}}\right] .
\end{aligned}\right.
$$

The soft finger is subjected to a combined bending. The stretch and compression can be neglected. With that, the hodograph $p^{\prime}(s)=\left(x^{\prime}(s), y^{\prime}(s), z^{\prime}(s)\right)$ of $p(s)$ (the FEA shape) meets with the following:

$$
p^{\prime}(s)=(\cos \phi(s) \sin \theta(s), \sin \phi(s) \sin \theta(s), \cos \theta(s)) .
$$

According to Eq. 7 and Eq. 10, the static equilibrium $(\mathrm{d} F(s)+f(s)=0)$ of the structure yields:

$$
F(s)=F_{e}-\rho g(s-L) \mathbf{y} .
$$

Considering that the distributed moment $M_{e x t}(s)=0$, and substituting Eq.11 and Eq.12 into Eq. 8, the following is obtained:

$$
\left\{\begin{aligned}
\theta^{\prime \prime}(s) & =\frac{F_{e_{z}} \sin \phi(s) \sin \theta(s)-\left(F_{e_{y}}-\rho g(s-L)\right) \cos \theta(s)}{(E I)_{e q_{x_{s}}}}, \\
\phi^{\prime \prime}(s) & =\frac{F_{e_{x}} \cos \theta(s)-F_{e_{z}} \cos \phi(s) \sin \theta(s)}{(E I)_{e q_{y_{s}}}}
\end{aligned}\right.
$$

with

$$
\left(F_{e_{y}}-\rho g(s-L)\right) \cos \phi(s) \sin \theta(s)=\sin \phi(s) \sin \theta(s) F_{e_{x}} .
$$

By considering FEA with multiple control inputs, the procedure can be generalized by introducing the convenient boundary condition at the portion linkages. In this paper, only two portions FEA have been considered (see Fig. 3), and the following holds:

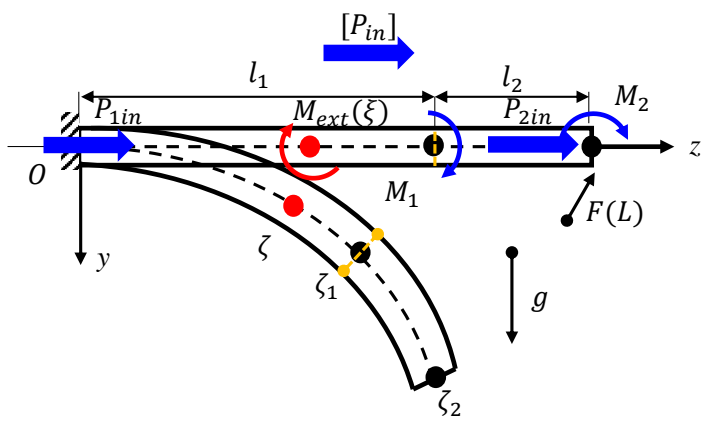

Fig. 3: Two serial FEA geometry

1) First phalange $\left(0 \leq s \leq \zeta_{1}\right)$

$$
p_{1}(s)=\int_{0}^{s} p^{\prime}(\xi) \mathrm{d} \xi
$$

2) Second phalange $\left(\zeta_{1} \leq s \leq \zeta_{2}\right)$

$$
p_{2}(s)=\int_{0}^{s} p^{\prime}(\xi) \mathrm{d} \xi+p_{1}\left(\xi_{1}\right),
$$

where the subscripts 1 and 2 stand for the first and second portion of the soft actuator, respectively.

\section{PH-EB BASED MODEL FOR SHAPE CONTROL}

To ease the modeling of the FEA shape, the advantageous features of parametric $\mathrm{PH}$ curves are combined with the EB model to control the FEA shape with external loads. For this purpose, the Bezier control points of the $\mathrm{PH}$ curve, representative of the FEA shape, can be expressed as functions of the actuation inputs $\left[P_{i n}\right]$ and the external loads.

\section{A. Forward Dynamic Model of FEA}

In this section, the FEA is modeled by parametric $\mathrm{PH}$ curves allowing to accurately describe their overall shape thanks to the knowledge of very few control points. To derive FEA shape kinematics in the Cartesian space, the following steps are considered:

\section{1) Polynomials functions settings of FEA PH curves}

The Quaternion form is chosen to simplify the writing in 3D. However, the length constraint in its Quaternion form is not easily comparing to its Complex form. Thus, a relation linking the Complex formulation and the Quaternion one of 
the hodograph is developed. Assume that $p_{c}$ and $p_{f}$ describe respectively the clamped and the tip positions of the FEA with $d_{c}$ and $d_{f}$, their respective directions under actuation input (eg. pressure effort). From Eq. 13 and Eq. 15, the pose of the robot tip can be computed regarding Eq. 10. Therefore, the following holds:

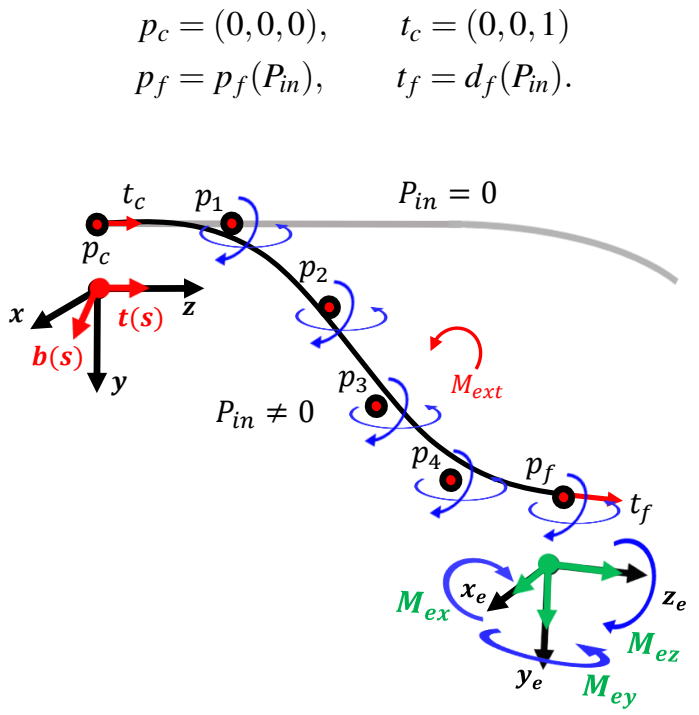

Fig. 4: $\mathrm{PH}$ virtual control points

Let us define $\zeta(0 \leq \zeta \leq 1)$, the normalized curvilinear coordinate along the neutral axis of the soft actuator with a constant length $L$. The following holds:

$$
p(\zeta)=(x(\zeta), y(\zeta), z \zeta)) ; \quad 0 \leq \zeta \leq 1
$$

First, the PH curve $p(\zeta)$ of the FEA and its hodograph $p^{\prime}(\zeta)=\left(x^{\prime}(\zeta), y^{\prime}(\zeta), z^{\prime}(\zeta)\right)$, namely the first derivative can be expressed in function of Quaternion polynomial $\mathcal{A}(\zeta)$ and its conjugate $A^{*}(\zeta)$, as follows [10]:

$$
\begin{aligned}
\mathcal{A}(\zeta) & =u(\zeta)+v(\zeta) \mathbf{i}+r(\zeta) \mathbf{j}+q(\zeta) \mathbf{k} \\
\mathcal{A}^{*}(\zeta) & =u(\zeta)-v(\zeta) \mathbf{i}-r(\zeta) \mathbf{j}-q(\zeta) \mathbf{k}
\end{aligned}
$$

where $\mathbf{i}, \mathbf{j}, \mathbf{k}$ represent the 3 quaternions basis elements of the set of quaternions $\mathbb{H}$. $u, v, r$, and $q$ defines the polynomial functions. In that case,

$$
p^{\prime}(\zeta)=\mathcal{A}(\zeta) \mathbf{i} \mathcal{A}^{*}(\zeta)
$$

Second, the same preceding PH curve $p(\zeta)$ and its hodograph $p^{\prime}(\zeta)$ can also be expressed in function of complex polynomials $\alpha(\zeta)$ and $\beta(\zeta)$ as follows [18]:

$$
\alpha(\zeta)=u(\zeta)+i v(\zeta), \quad \beta(\zeta)=q(\zeta)+i r(\zeta)
$$

To obtain the same $p^{\prime}(\zeta)$ expressed in (19) and (20), the quaternion polynomial $\mathcal{A}(\zeta)$ should be expressed in function of complex polynomials $\alpha(\zeta)$ and $\beta(\zeta)$, as follows :

$$
\mathcal{A}(\zeta)=\alpha(\zeta)+\mathbf{k} \beta(\zeta)
$$

where the imaginary unit $i$ is considered equivalent to the quaternion basis element $\mathbf{i}$.

2) Interpolation of end points using length constraints

The main objective is to determine the coefficients $\alpha_{m}$ and $\beta_{m}$ of the polynomial functions $\alpha$ and $\beta$, written with regard to
Bernstein form as described by (27). The parametric velocity polynomial $\Gamma(\zeta)$ is then expressed as follows:

$$
\Gamma(\zeta)=\left|p^{\prime}(\zeta)\right|=|\mathcal{A}(\zeta)|^{2}=|\alpha(\zeta)|^{2}+|\beta(\zeta)|^{2} .
$$

Integrating $p^{\prime}(\zeta)=\left(x^{\prime}(\zeta), y^{\prime}(\zeta), z^{\prime}(\zeta)\right)$ of (20) leads to end point displacements $\left(\Delta \mathbf{p}=p_{f}-p_{c}\right)$ satisfaction, described by equations (23) and (24) as follows:

$$
\begin{aligned}
& \int_{0}^{1}|\alpha(\zeta)|^{2}-|\beta(\zeta)|^{2} \mathrm{~d} \zeta=\Delta x . \\
& \int_{0}^{1} 2 \alpha(\zeta) \bar{\beta}(\zeta) \mathrm{d} \zeta=\Delta y+i \Delta z .
\end{aligned}
$$

The Fluidic actuator length constraint is calculated after integrating the equation (22) as follows:

$$
\int_{0}^{1}|\alpha(\zeta)|^{2}+|\beta(\zeta)|^{2} \mathrm{~d} \zeta=L
$$

After combining (23) with (25), the following relations are obtained:

$$
\begin{aligned}
& \int_{0}^{1}|\alpha(\zeta)|^{2} d \zeta=\frac{1}{2}(L+\Delta x), \\
& \int_{0}^{1}|\beta(\zeta)|^{2} d \zeta=\frac{1}{2}(L-\Delta x) .
\end{aligned}
$$

Note that, $\mathrm{PH}$ quintic curve can be generated by complex polynomials using Bernstein form:

$$
\begin{gathered}
\alpha(\zeta)=\alpha_{0}(1-\zeta)^{2}+2 \alpha_{1}(1-\zeta) \zeta+\alpha_{2} \zeta^{2}, \\
\beta(\zeta)=\beta_{0}(1-\zeta)^{2}+2 \beta_{1}(1-\zeta) \zeta+\beta_{2} \zeta^{2} .
\end{gathered}
$$

The canonical form $(\Delta \mathbf{p}=(1,0,0))$ should be adopted without loss of generalities. By substituting (27) into (24) and (26), the system obtained is consistent with (28).

\section{3) Calculation of Bernstein coefficients}

The end direction vectors are all formulated as $\mu$ proportional to the associated unit vectors, expressed in polar form (29) and (30). Thus, in order to have the first equation of (16) compatible with the remaining parts, the following relation is obtained:

$$
\begin{aligned}
& \left|\alpha_{0} \bar{\beta}_{2}+\alpha_{2} \bar{\beta}_{0}-3\left(\alpha_{0} \bar{\beta}_{0}+\alpha_{2} \bar{\beta}_{2}\right)\right|^{2} \\
= & {\left[12(L+1)-2\left(\left|\alpha_{0}\right|^{2}-\left|\alpha_{2}\right|^{2}\right)-\left|\alpha_{0}-\alpha_{2}\right|^{2}\right] } \\
& \cdot\left[12(L-1)-2\left(\left|\beta_{0}\right|^{2}-\left|\beta_{2}\right|^{2}\right)-\left|\beta_{0}-\beta_{2}\right|^{2}\right] .
\end{aligned}
$$

By specifying the unit end tangents in terms of polar, and azimuthal angles relative to $x$-axis as follows:

$$
\begin{aligned}
& t_{c}=\left(\cos \theta_{c}, \sin \theta_{c} \cos \phi_{c}, \sin \theta_{c} \sin \phi_{c}\right), \\
& t_{f}=\left(\cos \theta_{f}, \sin \theta_{f} \cos \phi_{f}, \sin \theta_{f} \sin \phi_{f}\right),
\end{aligned}
$$

the interpolation at end tangents yields :

$$
\begin{array}{ll}
\alpha_{0}=\mu c_{c} \exp \left(i \phi_{c}\right) \exp \left(i \psi_{0}\right), & \beta_{0}=\mu s_{c} \exp \left(i \psi_{0}\right), \\
\alpha_{2}=\mu c_{f} \exp \left(i \phi_{f}\right) \exp \left(i \psi_{2}\right), & \beta_{2}=\mu s_{f} \exp \left(i \psi_{2}\right),
\end{array}
$$

where $\psi_{0}$ and $\psi_{2}$ are free angular parameters [19] with

$$
\left(c_{c}, s_{c}\right)=\left(\cos \frac{1}{2} \theta_{c}, \sin \frac{1}{2} \theta_{c}\right),\left(c_{f}, s_{f}\right)=\left(\cos \frac{1}{2} \theta_{f}, \sin \frac{1}{2} \theta_{f}\right) .
$$


The use of (30) in (28) leads to the following form of the reduced bi-quadratic equation [20]:

$$
p\left(\mu^{2}\right)=c_{2} \mu^{4}+c_{1} \mu^{2}+c_{0} .
$$

4) Optimal position of the control points

With the above developments, the Quaternion coefficients $\mathcal{A}_{0,2}$ are calculated:

$$
\mathcal{A}_{0}=\alpha_{0}+\mathbf{k} \beta_{0}, \quad \mathcal{A}_{2}=\alpha_{2}+\mathbf{k} \beta_{2}
$$

In order to minimize the bending potential energy (35) during motion, the positive smaller root $\mu^{2}$ of (31) is considered. The end point displacement $\Delta \mathbf{p}$ allows us to define $\mathbf{d}$ as:

$$
\mathbf{d}=120 \Delta \mathbf{p}-15 \mu^{2}\left(d_{s}+d_{f}\right)+5\left(\mathcal{A}_{0} \mathbf{i} \mathcal{A}_{2}^{*}+\mathcal{A}_{2} \mathbf{i} \mathcal{A}_{0}^{*}\right) .
$$

Thus, the coefficient $\mathcal{A}_{1}$ is carried out through the end point displacement interpolation by the following expression:

$$
\mathcal{A}_{1}=-\frac{3}{4}\left(\mathcal{A}_{0}+\mathcal{A}_{2}\right)+\frac{\sqrt{|\mathbf{d}|}}{4} \frac{|\mathbf{d}| \mathbf{i}+\mathbf{d}}{|(|\mathbf{d}| \mathbf{i}+\mathbf{d})|} \exp \left(\psi_{1} \mathbf{i}\right)
$$

where $\psi_{1}$ is a free angular parameter. Therefore, the potential energy should be evaluated. For a curve-based kinematic model, it can be written in the canonical form as follows:

$$
E_{p}=\frac{(E I)_{e q}}{2} \int_{0}^{L}|\kappa(s)|^{2} \mathrm{~d} s, \quad \kappa(s)=\frac{\left|p^{\prime}(s) \times p^{\prime \prime}(s)\right|}{\left|p^{\prime}(s)\right|^{3}},
$$

with $\kappa(\zeta)$ the variable curvature along the FEA constant length. By imposing $\psi_{1}=0$, it is possible to determine the appropriate combination $\left(\psi_{0}, \psi_{2}\right)$ inducing a minimum value of the potential energy. The minimization of the potential bending energy of the FEA through PH formulation leads to five virtual control points that fully describe the related shape dynamics subject to an actuation input vector $\left[P_{i n}\right]$ :

$$
\begin{gathered}
p_{1}=p_{0}+\frac{1}{5} \mathcal{A}_{0} \mathbf{i} \mathcal{A}_{0}^{*}, \quad p_{2}=p_{1}+\frac{1}{10}\left(\mathcal{A}_{0} \mathbf{i} \mathcal{A}_{1}^{*}+\mathcal{A}_{1} \mathbf{i} \mathcal{A}_{0}^{*}\right), \\
p_{3}=p_{2}+\frac{1}{30}\left(\mathcal{A}_{0} \mathbf{i} \mathcal{A}_{2}^{*}+4 \mathcal{A}_{1} \mathbf{i} \mathcal{A}_{1}^{*}+\mathcal{A}_{2} \mathbf{i} \mathcal{A}_{0}^{*}\right), \\
p_{4}=p_{3}+\frac{1}{10}\left(\mathcal{A}_{1} \mathbf{i} \mathcal{A}_{2}^{*}+\mathcal{A}_{2} \mathbf{i} \mathcal{A}_{1}^{*}\right), \quad p_{5}=p_{4}+\frac{1}{5}\left(\mathcal{A}_{2} \mathbf{i} \mathcal{A}_{2}^{*}\right),
\end{gathered}
$$

with $p_{f}=p_{5}$ and $p_{c}=p_{0}$. The above control points $p_{k}(k=$ $1, \cdots, 5$ ), are all termed relative to the input pressure $\left[P_{i n}\right]$ (see Eq. 10 and Section III-A.3). The shape of the soft structure is recovered with:

$$
p\left(\zeta, P_{i n}\right)=\sum_{k=0}^{5} p_{k}\left(P_{i n}\right)\left(\begin{array}{l}
5 \\
k
\end{array}\right)(1-\zeta)^{5-k} \zeta^{k} .
$$

\section{B. Inverse Dynamic Model of FEA}

To set the real actuating pressure that drives the shape of the soft actuator, one needs to determine first the equivalent curvature $\kappa_{e q}$ of the finger portion to be actuated, and second, the corresponding control input $P_{i n}$ thanks to EB dynamics modeling (See Eq. 9).

Fig. 3 illustrates the kinematics of a 3 tubes-soft finger once actuated. The curvature $\kappa_{\alpha e q}$ in Eq. 9 should be regarded as the mean curvature exhibited by the considered portion of the structure. Note that the subscripts $\alpha=1,2$ account for the first and the second phalanges of the soft finger, respectively. In order to meet this requirement, the mean curvature $\kappa_{\alpha e q}$ of the desired shape, described using the PH curve, can be evaluated as described by Eq. 40 using the Darboux vector (Eq. 39).

The desired shape can be identified by knowing the control points $r_{k}$ [21].

$$
r(\zeta)=\sum_{k=0}^{5} r_{k}\left(\begin{array}{l}
5 \\
k
\end{array}\right)(1-\zeta)^{5-k} \zeta^{k} .
$$

Then, the variable curvature of Fig. 4, can be calculated as follows :

$$
\omega(s)=\kappa(s) \mathbf{b}(s)+\tau(s) \mathbf{t}(s), \quad \tau(s)=0 .
$$

Thus, the equivalent curvature $\kappa_{e q}$ is derived according to Eq. 40. Having done that, the equivalent curvature for each section of the soft finger is carried out with:

$$
\begin{aligned}
& {\left[\kappa_{1 e q}\right]=\frac{1}{l_{1}} \int_{0}^{\zeta_{1}} \omega(\xi) \mathrm{d} \xi,} \\
& {\left[\kappa_{2 e q}\right]=\frac{1}{l_{2}} \int_{\zeta_{1}}^{\zeta_{2}} \omega(\xi) \mathrm{d} \xi,}
\end{aligned}
$$

where $\zeta_{1}$ and $\zeta_{2}\left(\zeta_{2}=1\right)$ denote respectively the normalized curvilinear coordinates on the length of the soft finger. In accordance with Eq. 9 and Eq. 2, in the case of a 2-phalanges fingers (two actuated portions), it is possible to inverse the dynamics using the EB beam modeling as:

$$
\begin{aligned}
{\left[M_{2}\right]-\left(\sum P_{2 i n}\right) I_{2}(s) \kappa_{2 e q} } & =E_{2} I_{2}(s) \kappa_{2 e q}, \\
{\left[M_{1}\right]+\left[M_{2}\right]-\left(\sum P_{1 i n}\right) I_{1}(s) \kappa_{1 e q} } & =E_{1} I_{1}(s) \kappa_{1 e q},
\end{aligned}
$$

where $I_{\alpha}(s)=\operatorname{diag}\left(I_{\alpha_{x}}(s), I_{\alpha_{y}}(s), I_{\alpha_{z}}(s)\right), E_{\alpha}(\operatorname{dim}=1)$ are related to the portion $\alpha=1,2$ of the soft actuator. Eq. 41 describes a system of six equations with six unknowns, and thus allows us to define the actuation inputs of the robot corresponding to a prescribed shape.

\section{Materials ANd Methods}

This section describes the materials and validation method for the developed model used in dynamic shape control.

\section{A. Materials}

A single and multiple actuation inputs have been designed and manufactured for this purpose. They are made up of Agilus 30 soft material. Tensile tests using Instron (Mechanical testing machine) have been carried out to get the material properties. The results of these tests were fitted using Ansys 2019 on the consistency of Yeoh hyperelastic model for incompressible materials. See Fig. 5, Tab. I for description and parameters.

TABLE I: 2D FEA parameters

\begin{tabular}{l|l}
\hline FEA materials constant & FEA geometrical features \\
\hline$C_{1}=0.062 \mathrm{Mpa}$ & $L_{0}: 87 \mathrm{~mm}$ (single input) \\
$v=0.45$ & $L_{1}: 57 \mathrm{~mm}\left(1^{\text {st }}\right.$ phalange $)$ \\
$E=0.4 \mathrm{Mpa}$ & $L_{2}: 63 \mathrm{~mm}\left(2^{\text {nd }}\right.$ phalange $)$ \\
\hline
\end{tabular}




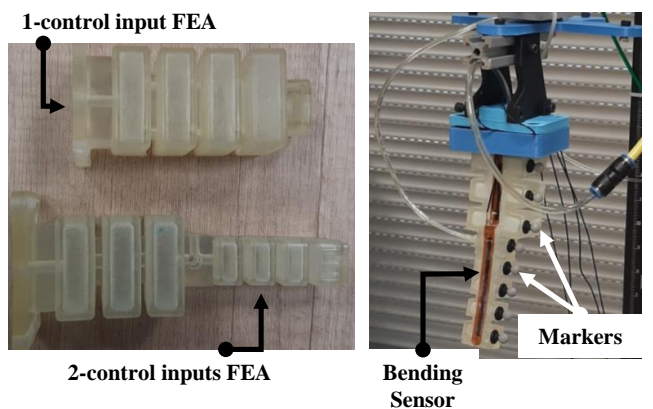

Fig. 5: Experiments Materials on 2D soft fingers

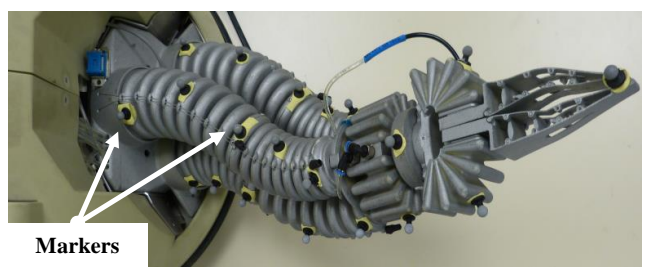

Fig. 6: CBHA continuum robot with its 6 actuator inputs

TABLE II: CBHA model parameters

\begin{tabular}{l|l}
\hline CBHA materials constant & CBHA geometrical features \\
\hline$\rho=1015 \mathrm{~kg} \cdot \mathrm{m}^{-3}$ & Backbone Length $L: 300 \mathrm{~mm}$ \\
$\nu=0.45$ & $L_{1}: 170 \mathrm{~mm}\left(1^{\text {st }}\right.$ section $)$ \\
$E=1.1 \mathrm{Gpa}$ & $L_{2}: 130 \mathrm{~mm}\left(2^{\text {nd }}\right.$ section $)$ \\
\hline
\end{tabular}

In addition, 3D shape control experiments on CBHA (see Fig. 6 and Tab. II ) have been performed.

During experiments, the Optitrack acquisition system has been used to capture shapes and displacements of the actuators equipped with markers. The accuracy of the Optitrack system is approximately $0.34 \mathrm{~mm}$. The bending sensors (Flexpoint Sensor System) were also embedded at the bottom of the in-extensible layer to assess the actuator shape in real time. The real object shape to be tracked is sensed using a 3Dcamera. The present approach has been investigated in the case of a static object. Therefore, only one image capture by the camera largely holds by following the protocols presented in section IV-B. For more insight, the parameters used for the experiments are given in the table III. The Festo Proportional air regulators (FESTO VPPM-6L-L-1-G18-0L6H-V1P) have been employed for the air pressure control, while the Simulink Dspace has been used for the real time acquisition and control system.

\section{TABLE III: Experiment test bench parameters}

\begin{tabular}{l|l}
\hline Hardware & Performances \\
\hline Optitrack System & $\begin{array}{l}\text { Mean 3D error: 0.348 } \mathrm{mm} \\
\text { Frequency: 120 FPS } \\
\text { Marker size (diameter): } 6.4 \mathrm{~mm}\end{array}$ \\
\hline Dspace controler & Frequency: $20 \mathrm{kHz}$ \\
\hline Air regulator & $\begin{array}{l}\text { Response time: } 0.5 \mathrm{~ms} \\
\text { Accuracy: } 2 \%\end{array}$ \\
\hline Bending sensors & Repeatability error: $0.3^{\circ}$ \\
\hline
\end{tabular}

FPS: Frame Per Second

\section{B. Methods}

This subsection deals with the inverse computation of the dynamics of the flexible finger. The objective is to define the actuation inputs such that they can control the overall shape in a non-contact case. The essential steps for the proposed approach are discussed as follows:

Extraction of the target object contour : The object contour is extracted for shape reconstruction of the soft finger, by computing the data images got from the 3D camera. The data are split into 2 parts for parallel grasping positioning, according to the geometry of the object.

Object shape extraction : A reference shape is identified using a PH control polygon (See Fig. 4) since the poses of the endpoints of the grasping process are known. This modeling enables adjustment of the control points poses for shape adaptability or accuracy issues.

Curvature computation : After having identified the shape to be tracked, the equivalent curvature as discussed by Eq. 40 is computed. This latter one is varying depending on the control polygon configuration.

Control inputs setting : The calculation of the dynamic inputs is defined by Eq. 41 for the studied classes of soft fingers. The input air pressure is converted to an analog signal and is provided to the Festo air regulator using the Simulink Dspace controller.

As described in the methodology, the validation scheme is given in Fig. 7. After extracting the object shape to be gripped, the reference shape of the soft gripper is obtained by $\mathrm{PH}$ modeling. Then, the geometrical features allowing to have the complex shape are estimated. The inverse model is used to determine the different control inputs to obtain the complex reference shape. Finally, these control inputs are applied to the real system. A comparison of the real data with those from the model simulation allows estimating the model deviations.

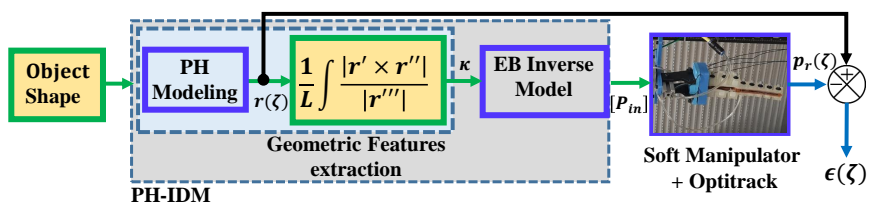

Fig. 7: PH and inverse EB Model validation scheme

\section{RESUlts AND Discussions}

The validation process consists of two main steps: In the first step, a simulation analysis (Section V-A) is performed where the outputs of the proposed PH-EB are compared to the shape of the object to be tracked. In the second step, an online analysis (Sections V-B and V-C) yields the real-world experiments.

\section{A. Numerical results of shape reconstruction}

In the following, the shape reconstructions for two types of objects are presented; one with a constant curvature describing a ball and another with a variable curvature (Fig. 8) describing a mango. In this figure, it is shown two soft fingers encapsulating the objects from each side for shape reconstruction and 
grasping tasks. The red contour legend represents the desired shape of the object extracted from the object contour. The blue curve with its control points describes the reconstructed shape, from the $\mathrm{PH}$ modeling allowing estimating by $\mathrm{EB}$ inverse dynamics, the corresponding desired actuation inputs. The grasping process is made on the $x y$-plane.

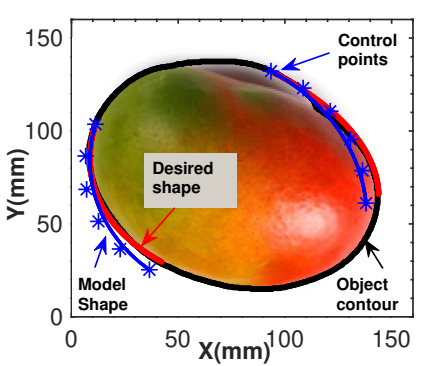

(a) Single input actuator

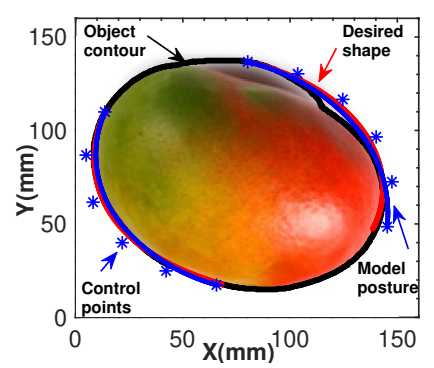

(b) Multiple inputs actuator
Fig. 8: Shape reconstruction with soft fingers

On Fig. 8a, the data using one control input actuator are plotted. Fig. 8b shows an accurate reconstructed shape (Blue legend) with two phalanges soft actuator and therefore, highlights the features of multiple actuation for shape control of the FEA. On Fig. 8a, the reconstruction of the object exhibits errors bigger than those observed in Fig. 8b. The errors observed in Fig. 8a can be explained by the complex shape geometry of the considered object. Indeed, a variable curvature is induced along the length of the desired shape. This is following the Eq. 11, where the actuation pressure input is computed using a constant curvature.

However, when experimenting with a constant curvature shape reconstruction described by a ball, the reconstructed shape of the ball (90 $\mathrm{mm}$ of diameter) using two phalanges appears less accurate compared to the case of one phalange, where a single actuator is considered. This observation can be explained by the difference in the arc length between the desired shape and the reconstructed one. These results show that for optimal shape reconstruction, it is necessary to find an optimal distribution of actuation inputs along the length of the soft fingers.

\section{B. Experimental validation on $2 D$ soft fingers}

Experimental tests of the PH-EB model-based control have been carried out on the soft fingers described previously [12]. They have also been compared to the ANSYS numerical modeling and real truncated shapes (right side) for the studied contour of Mango objects. The range of pressure at each actuator input varies between 0 and $55 \mathrm{kPa}$. Fig. 9a describes a shape tracking experiments with a single phalange. Only one set of data (right side) was chosen to investigate the shape tracking in the present contribution.

The Ansys Data analysis and the position of the Optitrack markers have also been described. To appreciate the quality of the analysis, Cartesian errors are analyzed along the soft actuator length. The error of the modeling relative to experiments has a maximum value of $8 \mathrm{~mm}(9.2 \%$ of the robot length). This value confirms the difficulties to meet the shape

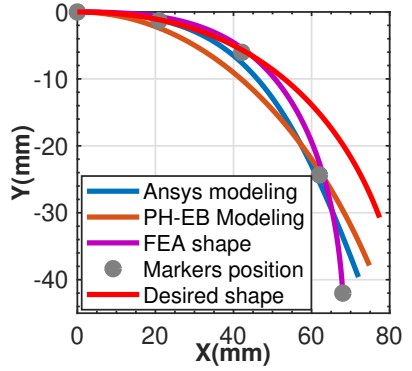

(a) Shape with a single input

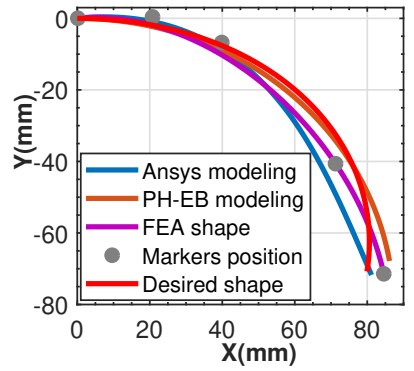

(b) Shape with multiple inputs
Fig. 9: Shape reconstruction from Mango

reconstruction using a single actuator input (single phalange), as explained in Section V-A. However, this error is coherent with $\varepsilon_{\max } \approx 8 \mathrm{~mm}$ ( $9.2 \%$ of the robot length), which describes the maximum error given by the experiments relative to the desired shape. This observation highlights the advantageous features of PH-EB modeling for soft continuum manipulator shape control.

From Fig. 9b, it is observed that the mean error between the PH-EB model and the desired shape has a maximum value close to $5 \mathrm{~mm}$ ( $4.2 \%$ of the robot length). It is smaller compared to that of Fig. 9a. This proves that in the case of the Mango, the performance of using multiple actuator inputs for accurate shape reconstruction. In addition, $\varepsilon_{\max } \approx 7 \mathrm{~mm}$ (5.83\% of the robot length) exhibits a biggest value at the soft fingertip.

\section{Experimental validation on $3 D$ CBHA continuum ma- nipulator}

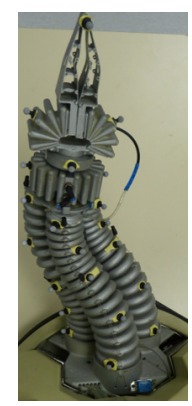

(a) Real soft continuum robot

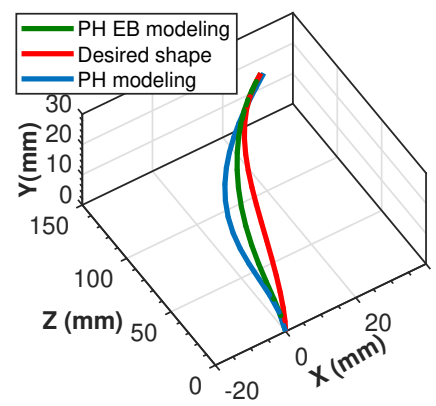

(b) Kinematics reconstruction
Fig. 10: 3D CBHA Shape in free case

For the 3D CBHA shape of Fig. 10a, a reference shape is tracked (red curve) in a free case. The PH modeling (blue color) described in [10] makes a shape kinematics reconstruction without considering the length of robot constraint. By observing the experiment results, a mean position error between the 400 points of the real robot shape and those given by the $\mathrm{PH}$ modeling is equal to $4.4 \mathrm{~mm}(1.5 \%$ of the robot length). Using the PH-EB modeling (green color), which respects the length constraint of the robot, the obtained mean error is about $2.3 \mathrm{~mm}$ ( $0.7 \%$ of the robot length). Furthermore, external efforts on the robot (Fig. 11) have been investigated in load cases by adding a mass of $200 \mathrm{~g}$ at the tip of the robot. For the same desired endpoint (tip point) 
as in the free case, a shape deformation occurs along the soft continuum manipulator. This deformation is kinematically reproduced by the $\mathrm{PH}$ modeling with fewer accuracy performances according to the desired endpoint. However, the PHEB modeling allows calculating the optimal actuator inputs (pressures) for accurate shape reconstruction and the desired endpoint tracking. Comparative performances between the $\mathrm{PH}$ modeling reconstruction [10] and the PH-EB modeling with adaptive reconstruction from actuator inputs are given in Table IV.

Fig. 11 presents the 3D shape kinematics while considering external load. The results of the experiments have been analyzed and showed for $\mathrm{PH}$ modeling reconstruction, indicating a mean error position for the shape of $12.3 \mathrm{~mm}(4.1 \%$ of the robot length) and of $11.2 \mathrm{~mm}(3.7 \%$ of the robot length) for the tip point tracking. After applying the PH-EB modeling, the mean error of the shape is about $7.1 \mathrm{~mm}(2.4 \%$ of the robot length) and the tip tracking error $3.8 \mathrm{~mm}(1.26 \%$ of the robot length).

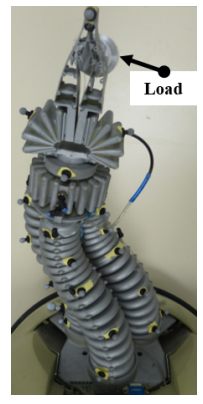

(a) Real soft continuum robot

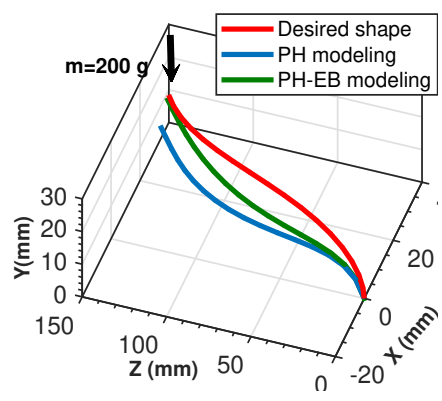

(b) Kinematics reconstruction
Fig. 11: CBHA Shape kinematics in case of external load

TABLE IV: Comparison results in case of external load

\begin{tabular}{c|c|c|c}
\hline Methods & SME & MTE & Time cost \\
\hline PH EB modeling & $7.1 \mathrm{~mm}(2.4 \%)$ & $3.8 \mathrm{~mm}(1.26 \%)$ & $0.0004 \mathrm{~s}$ \\
PH modeling & $12.3 \mathrm{~mm}(4.1 \%)$ & $11.2 \mathrm{~mm}(3.7 \%)$ & $0.00031 \mathrm{~s}$ \\
\hline
\end{tabular}

SME: Shape Mean Error, MTE: Mean Tip Error

\section{CONCLUSION}

In this paper, inverse dynamic modeling for $2 \mathrm{D}$ and $3 \mathrm{D}$ shape reconstruction of soft continuum robots based on the PH-EB approach is proposed. The modeling combines features of Euler-Bernouilli's beam mechanics and those of $\mathrm{PH}$ parametric curves with the macro and micro properties of the soft structure. It has been shown that Euler-Bernoulli's beam mechanics can be coupled with $\mathrm{PH}$ quintic to identify the relationship between the desired actuator inputs and the position of the finite virtual control points used for the shape reconstruction in the contact-free cases and external load cases. The results obtained show the coherence and relevance of the proposed approach to reconstruct complex-shaped objects in 2D and 3D with different types of soft continuum robots. Moreover, the developed approach allows implementing a form of enclosure grasping. It is expected that this modeling can be applied to various FEA, allowing performing the shape control. For future works, it is planned to extend the present work to the shape reconstruction of the FEA in dynamic motion.

\section{REFERENCES}

[1] D. Rus and M. Tolley, "Design, fabrication and control of soft robots," Nature, vol. 521, no. 7553, pp. 467-75, 2015.

[2] H. Mochiyama, E. Shimemura, and H. Kobayashi, "Shape control of manipulators with hyper degrees of freedom," The International Journal of Robotics Research, vol. 18, no. 6, pp. 584-600, 1999.

[3] C. Della Santina, R. K. Katzschmann, A. Bicchi, and D. Rus, "Modelbased dynamic feedback control of a planar soft robot: Trajectory tracking and interaction with the environment," The International Journal of Robotics Research, vol. 39, no. 4, pp. 490-513, 2020.

[4] C. Della Santina, A. Bicchi, and D. Rus, "On an improved state parametrization for soft robots with piecewise constant curvature and its use in model based control," IEEE Robotics and Automation Letters, vol. 5, no. 2, pp. 1001-1008, 2020.

[5] C. Della Santina, "The soft inverted pendulum with affine curvature," in 2020 59th IEEE Conference on Decision and Control (CDC). IEEE, 2020, pp. 4135-4142.

[6] S. H. Sadati, S. E. Naghibi, I. D. Walker, K. Althoefer, and T. Nanayakkara, "Control space reduction and real-time accurate modeling of continuum manipulators using ritz and ritz-galerkin methods," IEEE Robotics and Automation Letters, vol. 3, no. 1, pp. 328-335, 2017.

[7] S. H. Sadati, S. E. Naghibi, A. Shiva, B. Michael, L. Renson, M. Howard, C. D. Rucker, K. Althoefer, T. Nanayakkara, S. Zschaler, et al., "Tmtdyn: A matlab package for modeling and control of hybrid rigid-continuum robots based on discretized lumped systems and reduced-order models," The International Journal of Robotics Research, vol. 40, no. 1, pp. 296-347, 2021.

[8] A. L. Orekhov and N. Simaan, "Solving cosserat rod models via collocation and the magnus expansion," in 2020 IEEE/RSJ International Conference on Intelligent Robots and Systems (IROS). IEEE, 2020, pp. 8653-8660.

[9] F. Boyer, V. Lebastard, F. Candelier, and F. Renda, "Dynamics of continuum and soft robots: A strain parameterization based approach," IEEE Transactions on Robotics, 2020.

[10] I. Singh, Y. Amara, A. Melingui, P. Mani Pathak, and R. Merzouki, "Modeling of continuum manipulators using pythagorean hodograph curves," Soft Robotics, vol. 5, no. 4, pp. 425-442, 2018.

[11] S. Mbakop, G. Tagne, O. Lakhal, R. Merzouki, and S. V. Drakunov, "Path planning and control of mobile soft manipulators with obstacle avoidance," in 2020 3rd IEEE International Conference on Soft Robotics (RoboSoft). IEEE, 2020, pp. 64-69.

[12] S. Mbakop, G. Tagne, M.-H. Frouin, and R. Merzouki, "Interoperable models for dynamics and shape tracking of soft fingers," in 2021 IEEE 4th International Conference on Soft Robotics (RoboSoft). IEEE, 2021, pp. 199-206.

[13] M. Wiese, K. Rüstmann, and A. Raatzl, "Kinematic modeling of a soft pneumatic actuator using cubic hermite splines," in 2019 IEEE/RSJ International Conference on Intelligent Robots and Systems (IROS). IEEE, 2019, pp. 7176-7182.

[14] P. S. Gonthina, A. D. Kapadia, I. S. Godage, and I. D. Walker, "Modeling variable curvature parallel continuum robots using euler curves," in 2019 International Conference on Robotics and Automation. IEEE, 2019, pp. $1679-1685$.

[15] C. Wielgosz et al., "Bending and buckling of inflatable beams: some new theoretical results," Thin-walled structures, vol. 43, no. 8, pp. 11661187, 2005.

[16] Mihael Brojan, Tomaz Videnic, and Franc Kosel, "Non-prismatic nonlinearly elastic cantilever beams subjected to an end moment," Journal of Reinforced Plastics and Composites, vol. 26, no. 11, pp. 1071-1082, 2007.

[17] D. Trivedi, A. Lotfi, and C. D. Rahn, "Geometrically exact models for soft robotic manipulators," IEEE Transactions on Robotics, vol. 24, no. 4, pp. 773-780, 2008

[18] R. T. Farouki and T. Sakkalis, "Pythagorean hodographs," IBM Journal of Research and Development, vol. 34, no. 5, pp. 736-752, 1990.

[19] R. T. Farouki, M. al Kandari, and T. Sakkalis, "Hermite interpolation by rotation-invariant spatial pythagorean-hodograph curves," Advances in Computational Mathematics, vol. 17, no. 4, pp. 369-383, 2002.

[20] R. T. Farouki, "Existence of pythagorean-hodograph quintic interpolants to spatial g1 hermite data with prescribed arc lengths," Journal of Symbolic Computation, vol. 95, pp. 202-216, 2019.

[21] R. T. Farouki and T. Sakkalis, "Pythagorean-hodograph space curves," Advances in Computational Mathematics, vol. 2, no. 1, pp. 41-66, Jan 1994. 